\title{
Graphene based piezoresistive pressure sensor
}

\author{
Shou-En Zhu, ${ }^{1, a)}$ Murali Krishna Ghatkesar, ${ }^{1}$ Chao Zhang, ${ }^{2}$ and G. C. A. M. Janssen ${ }^{1}$ \\ ${ }^{1}$ Micro and Nano Engineering Lab, Department of Precision and Microsystems Engineering, \\ Delft University of Technology, Mekelweg 2, 2628CD Delft, The Netherlands \\ ${ }^{2}$ Kavli Institute of Nanoscience, Delft University of Technology, Lorentzweg 1, 2628CJ Delft, The Netherlands
}

(Received 30 January 2013; accepted 9 April 2013; published online 23 April 2013)

\begin{abstract}
We present a pressure sensor based on the piezoresistive effect of graphene. The sensor is a $100 \mathrm{~nm}$ thick, $280 \mu \mathrm{m}$ wide square silicon nitride membrane with graphene meander patterns located on the maximum strain area. The multilayer, polycrystalline graphene was obtained by chemical vapor deposition. Strain in graphene was generated by applying differential pressure across the membrane. Finite element simulation was used to analyze the strain distribution. By performing electromechanical measurements, we obtained a gauge factor of $\sim 1.6$ for graphene and a dynamic range from 0 mbar to 700 mbar for the pressure sensor. (c) 2013 AIP Publishing LLC [http://dx.doi.org/10.1063/1.4802799]
\end{abstract}

Graphene, a monoatomic thin $\mathrm{sp}^{2}$ bonded honeycomb carbon film, is the most robust material known. Since the discovery of graphene in $2004,{ }^{1}$ an enormous amount of effort has been invested in order to realize the application of graphene in prototype products such as flexible touch screens, ${ }^{2}$ high frequency transistors, ${ }^{3}$ and ultra-fast photodetectors. ${ }^{4}$ One of the promising fields for graphene application is micro electro mechanical systems (MEMS). Here, the extreme stiffness of graphene, with Young's modulus of $1 \mathrm{TPa},{ }^{5}$ excellent electrical conductivity compared to any other nanomaterials, ${ }^{6}$ super flexibility, and stretchability up to $20 \%{ }^{7}$ can be exploited. Furthermore, the resistivity of graphene varies linearly with strain. ${ }^{7,8}$ This piezoresistive effect is also observed in thin metal films and semiconductor films. The piezoresistive effect in those materials has been widely used in the MEMS smart sensor field, including strain gauges, cantilever force sensors, accelerometers, inertial sensors, and pressure sensors. ${ }^{9}$ By providing an easy and direct transduction, piezoresistive effect of graphene can be used to connect the mechanical and the electrical domains. At present various strain sensors based on graphene have been developed. ${ }^{7,8,10-13}$ However, the most important step for piezoresistive graphene, monolithic integration in silicon based MEMS, has not yet been reported.

In this letter, we present a graphene based piezoresistive pressure sensor based on standard semiconductor processes. See supplementary material for device fabrication details. ${ }^{14}$ A silicon nitride $\left(\mathrm{SiN}_{\mathrm{x}}\right)$ suspended membrane was used as the structural element in this pressure sensor. The mechanical properties of the $\mathrm{SiN}_{\mathrm{x}}$ membrane were measured by bulge testing. ${ }^{15}$ From the measurement of the center deflection as a function of the applied pressure, the stress-strain relation of the material can be obtained. ${ }^{16-18}$ The schematic of a membrane under test is shown in Fig. 1(a). When a differential pressure is applied, the force is uniformly distributed on top of the window, and the membrane undergoes deflection that is about two orders of magnitude larger than the membrane thickness. As illustrated in Fig. 1(b), the

\footnotetext{
${ }^{\text {a) }}$ Author to whom correspondence should be addressed. Electronic mail: shouen.zhu@tudelft.nl.
}

membrane deforms into a concave shape under differential pressure. The three dimension (3D) surface profile of the membrane was mapped by white light interferometer (WYKO NT3300 Optical Profiler). During the experiment, differential pressure ranging from 0 to $700 \mathrm{mbar}$ in steps of 50 mbar was applied across the membrane, while the out of plane deflection profile of the membrane was recorded (see Fig. 1(c)). The maximum out-of-plane deflections $\delta$ were fitted with respect to differential pressure $\mathrm{P}$ by using the analytical Eq. (1) $)^{17,18}$

$$
P=\frac{B_{1} t \sigma_{o}}{(a / 2)^{2}} \delta+\frac{B_{2} f(\nu) t E}{(a / 2)^{4}(1-\nu)} \delta^{3},
$$

where $B_{1}, B_{2}$ are dimensionless constants, $\sigma_{o}$ is the initial stress in the membrane, $a$ is the side-length of the membrane, $\nu$ is Poisson ratio, $f(\nu)$ is geometry function and the value was set to be $1-0.271 \nu$ in this report, $\mathrm{t}$ is the thickness of membrane, and $E$ is Young's modulus. The parameters used are listed in Table I. In order to map the strain of the $\mathrm{SiN}_{\mathrm{x}}$ membrane, a simulation model was created using the finite element package (COMSOL Multiphysics), where the fixed square support boundary condition was applied to the four edges. The maximum tensile strain of $\sim 0.25 \%$ occurred at the middle of each edge (normal strain perpendicular to the edge) as illustrated in Fig. 2(b), and the differential pressure in the model was set as 600 mbar. So, the locations with high tensile strain were selected for the placement of piezoresistive sensors. The strain of graphene was assumed to be same as the strain of the top of the $\mathrm{SiN}_{\mathrm{x}}$ membrane. In order to effectively increase the length of the resistor and the amount of total resistance, graphene was cut in a meandering pattern by e-beam lithography within the high strain area as shown in Fig. 2(a). Resistance changes were measured using a Wheatstone bridge circuit. The Wheatstone bridge consists of four resistors connected in a loop. An input voltage of $2.5 \mathrm{~V}$ is applied across junctions of two parallel circuits as shown in Fig. S2a in supplementary material. ${ }^{14}$ Voltage drop across the resistors forms the output. The graphene piezoresistor $R_{g}$ that varies with strain was represented by $R_{g}=R+\Delta R$, and $R$ was measured to be $74.3 \mathrm{k} \Omega$ (see Fig. S2b in supplementary material ${ }^{14}$ ). The other resistors 
(a)

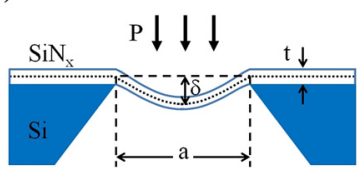

(c)

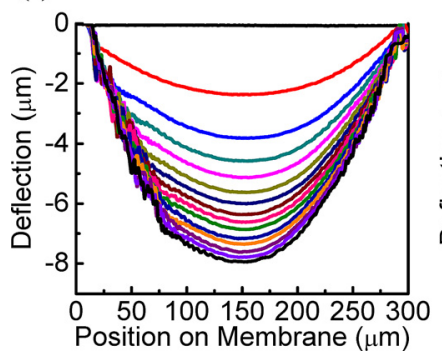

(b)

(d)
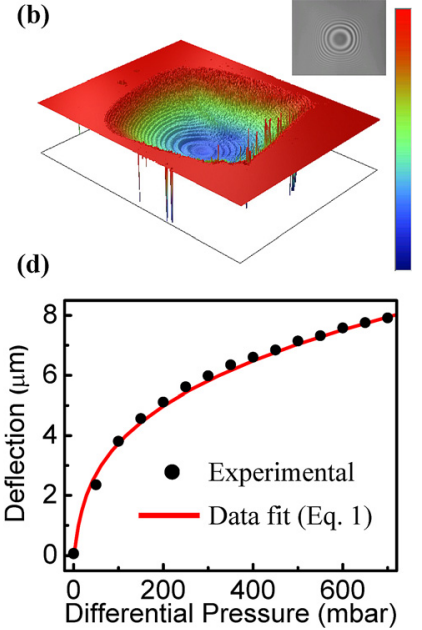

FIG. 1. (a) Schematic of suspended silicon nitride diaphragm under the applied differential pressure (the drawing is not to scale). (b) 3D deformation image of the $\mathrm{SiN}_{\mathrm{x}}$ membrane under differential pressure created by Optical Profiler (scale red to blue: 0 to $-6 \mu \mathrm{m}$ ). Inset shows typical optical interference fringes from the white light profilometer. (c) Deflection profile of the diaphragm under the differential pressure from 0 mbar to $700 \mathrm{mbar}$ (50 mbar per step). (d) Deflection versus differential pressure of $100 \mathrm{~nm}$ $\mathrm{SiN}_{\mathrm{x}}$ membrane. Experimental data were fitted (Eq. (1)) by the red line to estimate plane-strain elastic modulus presented in Table I.

$R_{1}, R_{2}$, and $R_{3}$ were tuned to be identical in value with the unstrained graphene resistor $R$ and located on a rigid Printed Circuit Board (PCB). The output voltage is linearly proportional the input voltage $V_{\text {in }}(2.5 \mathrm{~V})$, according to Eq. (2)

$$
V_{\text {out }}=V_{\text {in }}\left(\frac{R_{2}}{R_{1}+R_{2}}-\frac{R_{3}}{R_{3}+R_{g}}\right)=V_{\text {in }}\left(\frac{\Delta R}{2 R+\Delta R}\right) .
$$

In our measurement, the measured resistance variation of graphene was less than $1 \%$. The output voltage was quasilinearly rising in proportion to $\Delta R / R$. The reversible performance of the graphene piezoresistor was observed by switching the pressure in 22 cycles of ON/OFF test (Fig. 2(c)), which shows little degradation. Closer details due to pressure switching during each cycle are illustrated in Fig. 2(d). Each switching cycle was performed by turning vacuum pump on/off with fixed 600 mbar differential pressure. The $\sim 5 \mathrm{mV}$ transition, which is equal to $0.4 \%$ of resistance change, was reached in $0.9 \mathrm{~s}$. While turning off the pump, the strain was fully relaxed after $5.6 \mathrm{~s}$ due to the slow venting of the gas line. Measurements were also made at different differential pressures across the membrane as shown in Fig. 3(a). The output intensity increased in distinct steps as the tensile strain applied on graphene piezoresistor was

TABLE I. Properties of square $\mathrm{SiN}_{\mathrm{x}}$ membrane.

\begin{tabular}{|c|c|c|}
\hline LPCVD SiN $_{x}$ membrane & Value & Reference \\
\hline Thickness (nm) & 100 & \\
\hline Length $(\mu \mathrm{m})$ & 280 & \\
\hline Residue stress (MPa) & 50 & \\
\hline Constant $B_{1}$ & 3.45 & 18 \\
\hline Constant $B_{2}$ & 1.994 & 18 \\
\hline Poisson's ratio & 0.22 & 19 \\
\hline Young's modulus (GPa) & 200 & 19 \\
\hline
\end{tabular}

(a)

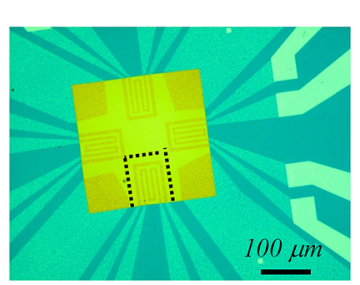

(b)

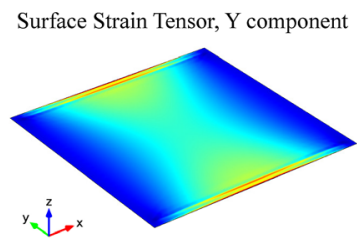

(c)

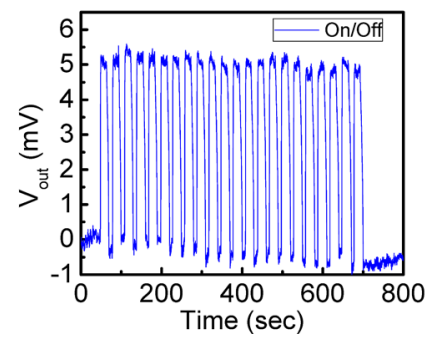

(d)

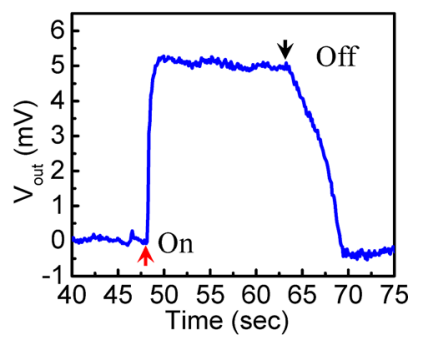

FIG. 2. (a) Optical microscope image of graphene piezoresistors on silicon nitride square membrane (the dotted lines highlight the device used for the measurement). (b) Y component of surface strain tensor, the entire surface shows tensile strain under differential pressure. The maximum strain occurs at the center of each edge (green: $\sim 0.25 \%$ strain; blue: $\sim 0 \%$ strain). The $\mathrm{X}$ component of the tensor shows same behavior as $\mathrm{Y}$ component, but $90^{\circ}$ rotated. (c) Output voltage versus time during cycling test with switching ON/OFF 600 mbar differential pressure. (d) One typical cycle in (c) with $15 \mathrm{~s}$ ON $15 \mathrm{~s}$ OFF under $600 \mathrm{mbar}$ differential pressure (ON/OFF indicates the switching of the valve for vacuum).

increased. These well-defined steps show the immediate response of the piezoresistive effect to the deformation of membrane. At $247 \mathrm{~s}$, the valve of the gas pump was closed. After slow venting of $15 \mathrm{~s}$, the output signal returned to $0 \mathrm{mV}$. This reverse (restore) time was determined by the response of the venting speed and the manual control when adjusting the differential pressure, and so the actual response time is anticipated to be faster. The ability to monitor the step of pressure demonstrates the accuracy of graphene piezoresistive effect for application of pressure sensor. The output voltages as function of applied differential pressure were also plotted in Fig. 3(b). It is clear that a good linearity was observed up to $500 \mathrm{mbar}$, and the sensor sensitivity $\sim 8.5 \mathrm{mV} /$ bar was obtained by fitting the slope. The standard deviation was less than $0.13 \mathrm{mV}$, which is $\sim 2.2 \%$ with respect to its dynamic range, i.e., $15 \mathrm{mbar}$ at $700 \mathrm{mbar}$ full scale. The pressure sensor working range, accuracy, and sensitivity can be further increased by changing the membrane thickness, optimizing graphene location and sensing wire pattern, and designing low noise electronics suitable for graphene. Finally, the gauge factor $\mathrm{G}$ of the graphene piezoresistor can be estimated from the change in resistance related to the applied strain. $G=\frac{\Delta R / R}{\Delta L / L}=\frac{\Delta R / R}{\varepsilon}=\sim 1.6$, which is in agreement with former reports, e.g., exfoliated graphene $\sim 1.9$ with $\sim 3 \%$ strain, $^{12}$ CVD graphene $\sim 6.1$ with $1 \%$ strain, ${ }^{8}$ and graphene ripples $\sim 2$ with an applied strain up to $30 \% .{ }^{11}$ This demonstrates the feasibility of an accurate pressure sensor comparable to the metal based strain gauge.

In conclusion, a graphene based piezoresistive pressure sensor was developed by integrating graphene resistors on a $\mathrm{SiN}_{\mathrm{x}}$ membrane. The characteristics of graphene piezoresistive effect and the sensitivity as function of applied differential pressure demonstrated the high performance of graphene 
(a)

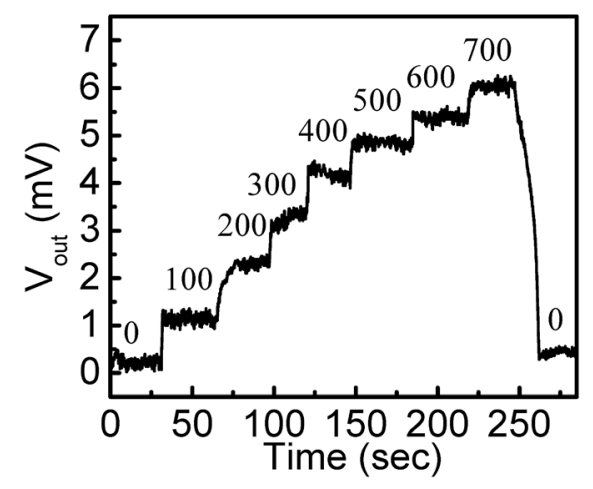

(b)

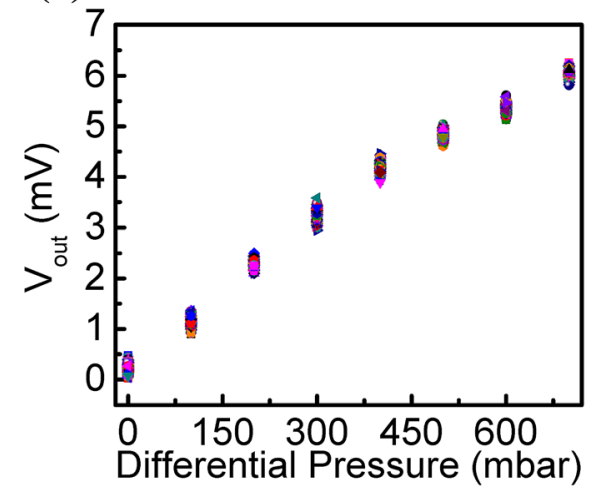

FIG. 3. (a) Dynamic output voltage versus time under differential pressure with step increase from 100 mbar to 700 mbar (100 mbar per step). (b) Output voltage as function of the differential pressure. for pressure sensor application. The sensitivity can be further improved by integrated full Wheatstone bridges based on graphene. This semiconductor compatible and controllable fabrication processes can pave a route for graphene based piezoresistive MEMS devices.

We acknowledge V. E. Calado for e-beam lithography and R. Luttjeboer, J. van Driel, M. Langelaar, H. Jansen, and P. van Holst for discussions and technical support. We acknowledge the financial support from the Young Wild Idea Grant of the Delft Centre for Materials. This work is part of the research program of the Foundation for Fundamental Research on Matter (FOM), which is part of the Netherlands Organisation for Scientific Research (NWO).

${ }^{1}$ K. S. Novoselov, A. K. Geim, S. V. Morozov, D. Jiang, Y. Zhang, S. V. Dubonos, I. V. Grigorieva, and A. A. Firsov, Science 306, 666 (2004).

${ }^{2}$ S. Bae, H. Kim, Y. Lee, X. Xu, J.-S. Park, Y. Zheng, J. Balakrishnan, T. Lei, H. R. Kim, Y. I. Song, Y.-J. Kim, K. S. Kim, B. Özyilmaz, J.-H. Ahn, B. H. Hong, and S. Iijima, Nat. Nanotechnol. 5, 574 (2010).

${ }^{3}$ Y.-M. Lin, C. Dimitrakopoulos, K. A. Jenkins, D. B. Farmer, H.-Y. Chiu, A. Grill, and P. Avouris, Science 327, 662 (2010).

${ }^{4}$ T. Mueller, F. Xia, and P. Avouris, Nat. Photonics 4, 297 (2010).

${ }^{5}$ C. Lee, X. Wei, J. W. Kysar, and J. Hone, Science 321, 385 (2008).
${ }^{6}$ J.-H. Chen, C. Jang, S. Xiao, M. Ishigami, and M. S. Fuhrer, Nat. Nanotechnol. 3, 206 (2008).

${ }^{7}$ K. S. Kim, Y. Zhao, H. Jang, S. Y. Lee, J. M. Kim, K. S. Kim, J.-H. Ahn, P. Kim, J.-Y. Choi, and B. H. Hong, Nature (London) 457, 706 (2009).

${ }^{8}$ Y. Lee, S. Bae, H. Jang, S. Jang, S.-E. Zhu, S. H. Sim, Y. I. Song, B. H. Hong, and J.-H. Ahn, Nano Lett. 10, 490 (2010).

${ }^{9}$ A. A. Barlian, W. Park, J. R. Mallon, Jr., A. J. Rastegar, and B. L. Pruitt, Proc. IEEE 97, 513 (2009).

${ }^{10}$ S.-H. Bae, Y. Lee, B. K. Sharma, H.-J. Lee, J.-H. Kim, and J.-H. Ahn, Carbon 51, 236 (2013).

${ }^{11}$ Y. Wang, R. Yang, Z. Shi, L. Zhang, D. Shi, E. Wang, and G. Zhang, ACS Nano 5, 3645 (2011).

${ }^{12}$ M. Huang, T. A. Pascal, H. Kim, W. A. Goddard, and J. R. Greer, Nano Lett. 11, 1241 (2011).

${ }^{13}$ S.-E. Zhu, R. Shabani, J. Rho, Y. Kim, B. H. Hong, J.-H. Ahn, and H. J. Cho, Nano Lett. 11, 977 (2011).

${ }^{14}$ See supplementary material at http://dx.doi.org/10.1063/1.4802799 for device fabrication and characterisation.

${ }^{15}$ J. W. Beams, Structure and Properties of Thin Films (John Wiley and Sons, NY, 1959), pp. 183-192.

${ }^{16}$ J. F. Creemer, S. Helveg, P. J. Kooyman, A. M. Molenbroek, H. W. Zandbergen, and P. M. Sarro, J. Microelectromech. Syst. 19, 254 (2010).

${ }^{17}$ M. G. Allen, M. Mehregany, R. T. Howe, and S. D. Senturia, Appl. Phys. Lett. 51, 241 (1987).

${ }^{18}$ D. Maier-Schneider, J. Maibach, and E. Obermeier, J. Microelectromech. Syst. 4, 238 (1995).

${ }^{19}$ K. B. Gavan, H. J. R. Westra, E. W. J. M. Van der Drift, W. J. Venstra, and H. S. J. Van der Zant, Microelectron. Eng. 86, 1216 (2009). 